\title{
Parametrised Design of a Cutting Device Used for High Tibial Osteotomy
}

\author{
Nicolae Florin Cofaru ${ }^{1, *}$, Ileana Ioana Cofaru ${ }^{1}$, Radu Emanuil Petruse ${ }^{1}$, Milan Rackov $^{2}$, \\ Mirko Blagojevic ${ }^{3}$ and Nenad Marjanovic ${ }^{3}$ \\ 1 "Lucian Blaga" University of Sibiu, Faculty of Engineering, Str.Emil Cioran Nr.4, 550025 Sibiu, \\ Romania \\ ${ }^{2}$ University of Novi Sad, Faculty of Technical Sciences, Trg Dositeja Obradovića 6, 21000 Novi Sad, \\ Serbia \\ ${ }^{3}$ University of Kragujevac, Faculty of Engineering, 34000 Kragujevac, Sestre Janjic 6, Serbia
}

\begin{abstract}
In this paper we present a design method for a modular device used in different types of HIGH TIBIAL OSTEOTOMY surgical procedures. The requirements, necessities and functions of this modular device are presented in the first part of the article. An important aspect is the fact that the device must be designed in a manner which permit to use it for all types of HIGH TIBIAL OSTEOTOMY: opening wedge osteotomies, closing wedge osteotomies, medial osteotomies, lateral osteotomies, uniplanar osteotomies or biplanar osteotomies. This fact is possible using modular elements for the main parts of the devices. The researches could be the starting point for others researches regarding the appropriate materials and the possibility to execution customized several parts of the modular device using Additive Manufacturing facilities.
\end{abstract}

\section{Introduction}

In this research we design a specialized modular device used for High Tibial Osteotomy (HTO), a surgical intervention which corrects the axial deviations of the human's leg.

The leg's mechanical axis (Figure 1.) is a line which unites three key elements: the middle of the hip's articulation, the middle of the knee's joint and the middle of the ankle's articulation. This line is comprised from 2 segments corresponding of the mains bones of the leg: tibia and femur. A suitable bones position is achieved when the two segments mentioned above are collinear. If this condition is not fulfilled, an axial deviation of the lower member appears, with significant consequences on the health and wellbeing of the patient. In Figure 1b a correctly aligned articulation is depicted with the green line versus a deviated axis shown with the red line.

An important cause of this axial deviation is the ghonartrosis, a disease characterised by the wear of the articular cartilage. The cartilage's wear is in general unicompartimental. This wear provokes an abnormal knee load which inevitably leads to an axial deviation. Once this deviation emerges, the worn cartilage is loaded even more, which will result in a higher axial deviation.

\footnotetext{
* Corresponding author: nicolae.cofaru@ulbsibiu.ro
} 
For example, an axial deviation with only 10 degrees, doubles the loads in applied on the knee's joint. Is very important to interrupt this vicious circle and the best way for doing so is the High Tibial Osteotomy surgical intervention.
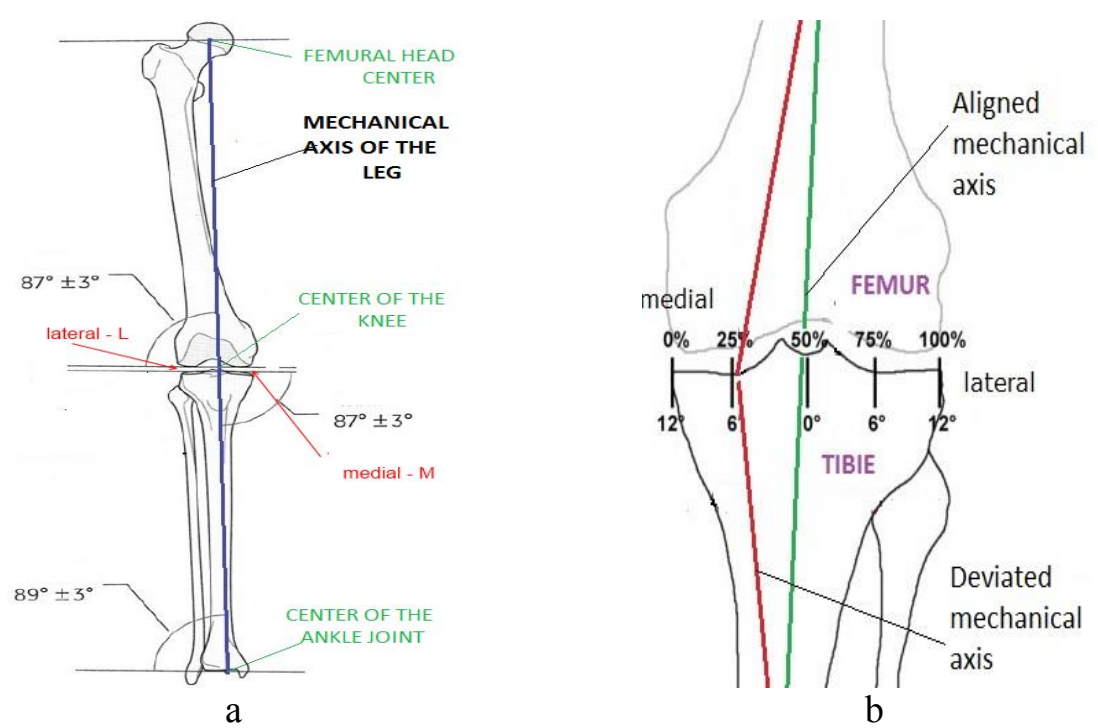

Fig. 1. Mechanical axis (aligned and deviated) of the human's lower member [1,5]

High Tibial Osteotomy imply creating a cut in the shape of a wedge on the lateral or median surface of the tibia and perform a rotation movement which will realign the leg's mechanical axis. In consequence, a normal load on the leg's joint will be displaced with diminished tensions on the affected area.
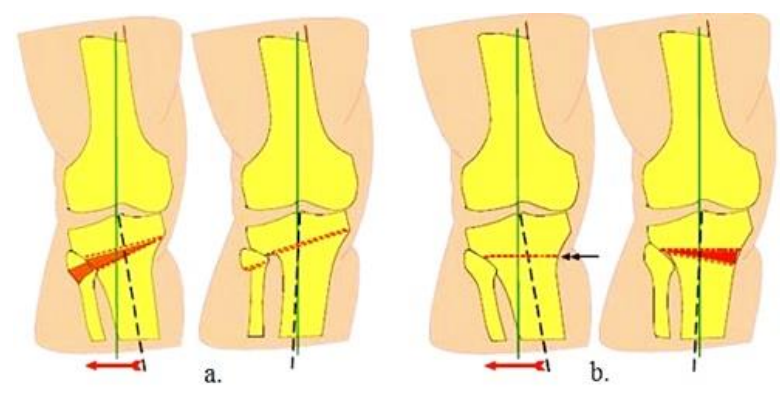

Fig. 2. High tibial surgeries: a -closing osteotomy, b -opening osteotomy [4]

Figure 2. depicts both osteotomy possibilities with a closing wedge and with an opening wedge. $[1,3,4,6]$. In order two achieved a Closing High Tibial Osteotomy the wedge is obtained with two cuts executed in general from the side of the tibia. The wedge can be eliminated, and the resulted surfaces are overlaid one over the other resulting a corresponding alignment of the mechanical axis (Figure 2a). In the case of an Opening High Tibial Osteotomy, the wedge is created with only one cut from the medial side of the tibia. The height of the wedge is determined by the correction angle required to fix the axial deviation (Figure 2b). 
In order to obtain a precise realignment of the bone, accurate geometrical and dimensional planning is required. For this purpose, we designed a modular specialized device which increase the accuracy and the precision of this surgical procedure.

\section{Parametrised design of the modular cutting device}

In the design process we adopted a generalized approach keen obtaining a high precision for the osteotomy wedge's peak (called CORA- centre of rotation of angulation). By using this modular device, we intended to aid the HTO procedure, thus increasing the precision. The generalized approach consists in a modular design which enables the device to be used for all kind of the possible High Tibial Osteotomies (closing, opening, medial, lateral, uniplanar, biplanar)

Taking in account the fact that the cuts required for the osteotomy wedges are three dimensional, to increasing accuracy of the HTO is obviously that a dedicated device is absolutely necessary

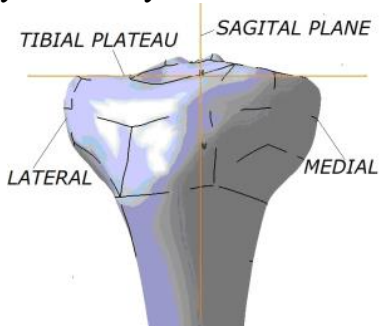

a.

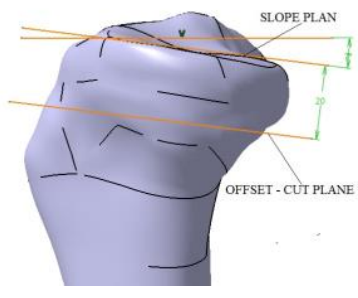

c.

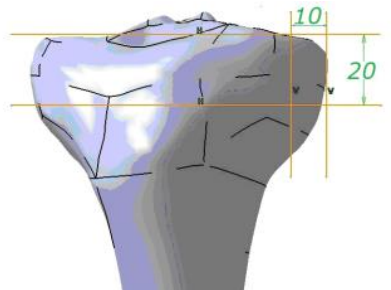

b.

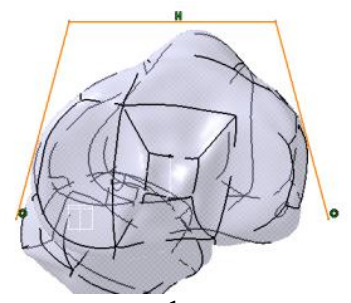

d..

Fig. 3. Geometrical reference elements used the device's design [5].

To obtain the constructive solutions for the device, the first step is to develop an orientation and fixture scheme for the tibia which enables the mechanical operations required for the HTO. For this goal, the next references planes will be taking in account (Figure 3.): the sagittal plane of the tibia, the tangent plane of the tibial condyles and the vertical tangent planes, of the medial and lateral cortical areas of the tibia (Figure 3a.). Using these geometrical references, the position of execution of CORA can be established (Figure 3b.). The CORA position was established in frontal plane. Because the tibia surface of the condyle has an inclination to the posterior with approximately 7-9 degrees (Figure. 3c.) it is necessary to tilt all the cutting planes at 7 to 9 degrees. From orientation point of view and by the tibia's geometrical configuration, we determined that the orientation surface is suitable to fit a short prism (Figure 3d).

To validate our design and to be sure that we have a feasible solution, we studied speciality articles and patents on this topic [7-11]. In order to find the best constructive solutions for the modular device, we established the main functions which the device must to meet in order to be operative:

- To offer the possibility to rigidly fix the device in the right position on the tibia

- To be able to tune the dimensional and geometrical position of the CORA point

- To be able to drill a stress relief hole with different diameters in the CORA point 
- To offer guiding slots which will enable precise cuts

- To be able to adjust the HTO correction angle

- To be able to assure the cuts convergency in the CORA point

Taking in account by the functions that we identified, the constructive solutions for the subassemblies of the device resulted.

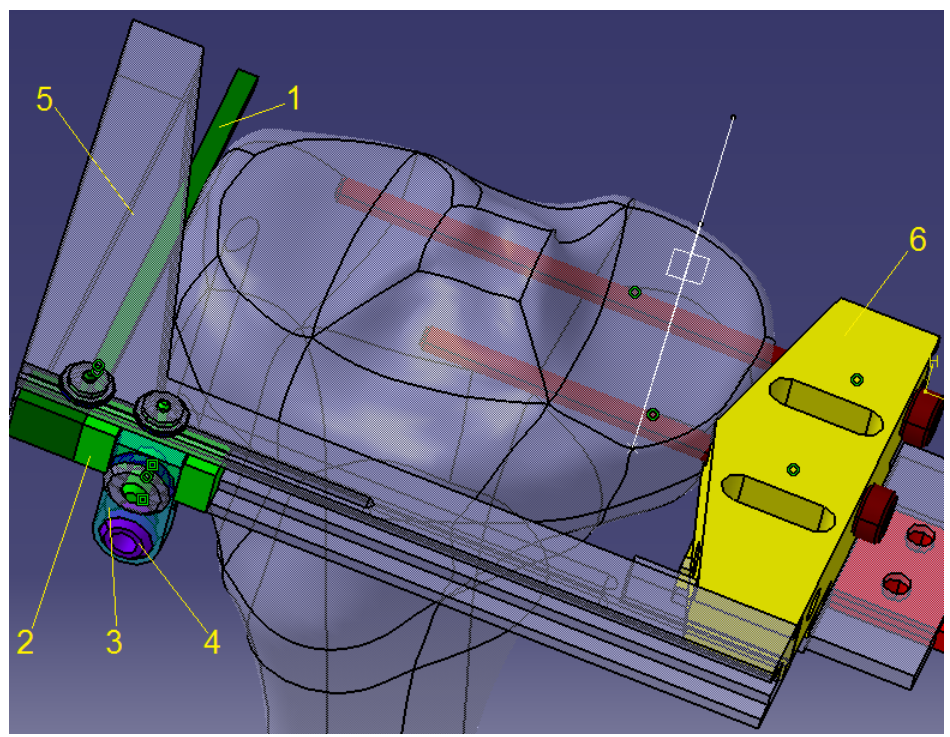

Fig. 4. Modular device - the assembly destined for execution of the CORA

For the orientation and fixation of the bone (tibia) our constructive solution consists from 2 parts: a double prism (6 from figure 4 ) and a mobile prism (5 from figure 4 ). These two parts are assembled and orientated on the tibia keeping in mind the condition to be tangent of the surface of the tibia condyle and taking in account the inclination of 7-9 degree as it resulted from Figure 3d. These two prisms have a modular construction, aspect which will be detailed in the next paragraph.

After the device is securely fixed on the tibia the first step is to assure the position required to drill the CORA stress relief hole. To achieve this, the lateral palpate pin (1 from figure 4) touches the lateral cortical bone surface. The slider (2 from figure 4 ) is brought in contact with the palpate pin. Both these components are able to perform linear movements through the mobile prism (5 from figure 4) adjustment channel. Considering that the palpate pin is in the right position, when they are in contact it means that little shaft situated in the medial plane of the slider is positioned at $8 \mathrm{~mm}$ to lateral surface of the tibia. For a different position (larger than $8 \mathrm{~mm}$ ) it is possible a controlled movement of the slider. In this step the positioning of the CORA in relation to the lateral cortical surface of the tibia is assured. In order to make the orientation in relation to the tibial plateau the bush support plate 3 are used. This plate has also designed in order to assure different distances from CORA to the tibial plateau.

In the assembly presented in figure 4, the bush support plate (no. 3) assures a $20 \mathrm{~mm}$ distance between the axis of the CORA point and the surface of the tibia condyle. For other distances which the surgical procedure may require different bush support plates with corresponding lengths can be added. For different diameters of the CORA stress relief hole a set of calibrated drill bushes (no.4) is available. These drill bushes are adjustable on the bush support plate (no.3). The hole is obtained with guided drilling through the drill bush. 
The modular device in this assembly way assure the first four functions regarding orientation, fixation of the tibia in device and precise manufacturing of the CORA.

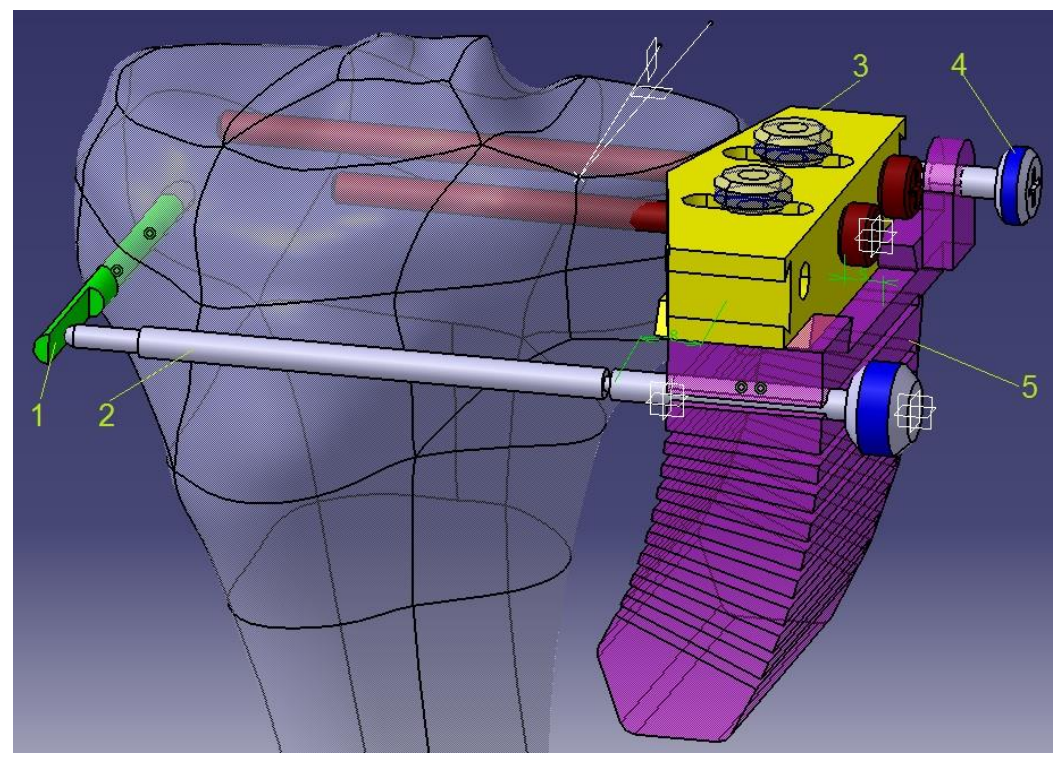

Fig. 5. Modular device destined for execution of the HTO cutting

After the execution of the CORA hole the previous parts of the device are demounted excepted the double prism with its fixation elements. On this prism the cutting guide (5 from figure 5) is assembled. This guide has radial channels disposed from 2 to 2 degrees. The superior channel is parallel to the superior surface of the device and it has a hole with the axis coincident with the same surface and perpendicular with the CORA stress relief hole's axis. Through this hole the control rod (2 from figure 5) is positioned. The control rod is pushed until it touches the planar face of the notched pin (1 from figure 5$)$ which is fixed in the CORA stress relief hole. Both elements (1 and 2) have a very important role in establishing the position of the cutting guide in relation to CORA and to assure a convergence of the cutting slots in the CORA point. For this convergence the position of the cutting guide in horizontal plane is critical as well. This positioning is fulfilled using the control rod (no. 2) which is assembled in the cutting guide like in Figure 5. When the tip of the rod touches the notched pin and the cutting body (no. 5) is inserted on the rod, the CORA point is the centre of the circle onto which are oriented the radial cutting guidance channels. To achieve this position, the positioning screw (no. 4) is used. In order to conserve this position, the screws (no. 3) is used.

Now the guide cutting is in working position and the notched pin (no. 1) and the control rod (no. 2) can be extracted. Taking into account the type of the HTO which is required the necessary cuttings can be done.

In the presented situation, the cutting guide is designed for the closing HTO which requires the execution of two cuts. Due to its modular design, the device can be easily configured to perform opening, uniplanar or biplanar osteotomies only by changing the cutting guide body.

\section{Modular design for a high degree of adaptability}

One of the most important features on which we focused during the design process of this surgery device was to have multiple surgical applications. First of all, the device must to be able to be used for all types of osteotomies: closing, opening, medial, lateral, uniplanar and 
biplanar HTO. Also, for each type of osteotomy the elements of the device have a modular and generalised design which can be geometrical and dimensional customized.

To execute the lateral osteotomy, the cutting is initiated from the lateral area of the tibia and when the cutting starts from the medial area, the osteotomy is a medial one. In order to make both operations with the same device, the orientation elements of the tibia, must be mounted in relation with the medial or lateral side of the bone. The constructive solution which takes into account this aspect was a symmetrical construction in relation with the medial and vertical plane of both prisms (red planes): the mobile prism and double prism (Figure 6). For the same reason the geometrical configuration of the second prism is doubled.
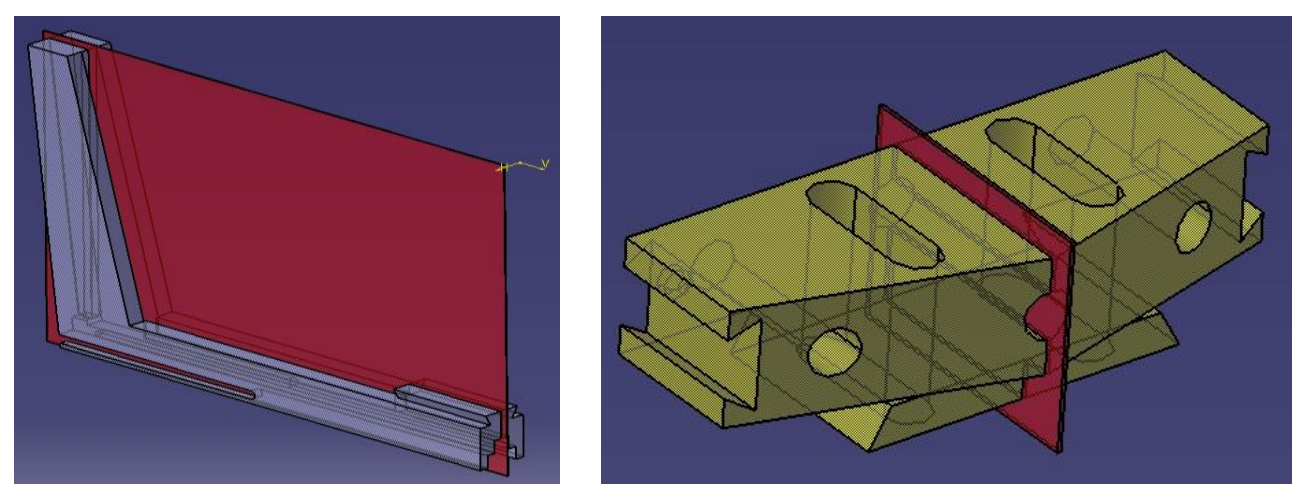

Fig. 6. Mobile prism and double prism - symmetrised constructions

In order to satisfy two of the device's the functional characteristics: the existence of a system which enables the dimensional and geometrical adjustments of the CORA point position and the possibility to drill a stress relief hole with different diameters in the CORA point, we designed two modular parts which can be customized depending on the surgery's requirements. These components are the bush support plate, and the guiding bush (Figure 7).

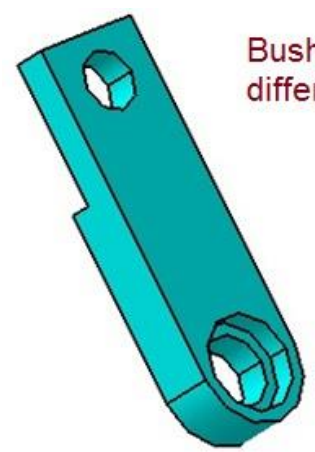

Bush support plates different dimensions

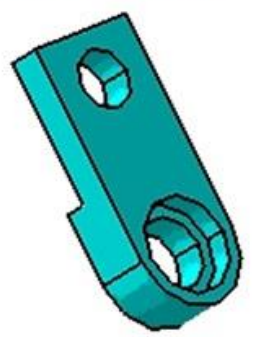

Guiding bush -

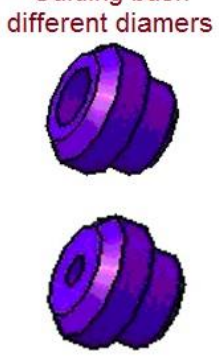

Fig. 7. Customization for CORA positions and diameters

Taking in account the usual dimensions for the distances between CORA and tibial plateau and usual diameters for CORA hole, kits of bush support plates and guiding bushes are manufactured. 
The component designed for the next functions which the device has to fulfil: to offer guiding slots which will enable precise cuts and to be able to adjust the HTO correction angle, is the cutting guide.
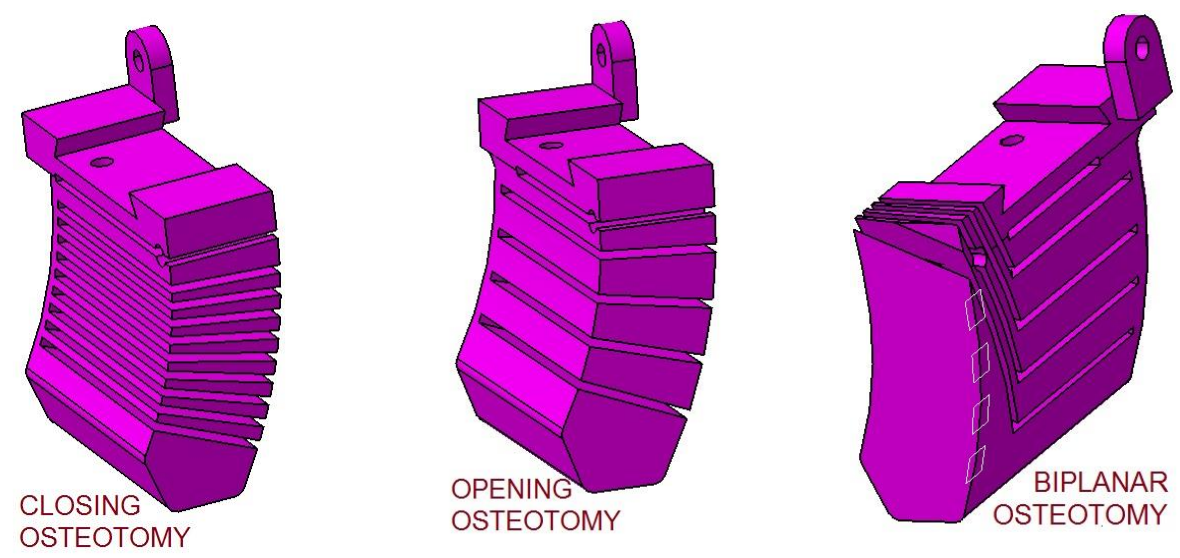

Fig. 8. Customization cutting guide for different types of the osteotomies

Every cutting guide depicted in figure 8 is fixed on the device in a similar manner because the superior surfaces destined for orientation and fixation in device are the same.

The functioning of the cutting guide destined for closing HTO was presented in the above paragraphs. For the opening osteotomy surgery, as mentioned above, a single cut is necessary, with a certain inclination towards the tibia surface of the condyle. For this reason, the cutting bodies have a similar configuration, the only difference consist in the way the radial channels are disposed. For the uniplanar opening osteotomy, the cutting channels are disposed in 3-4 positions necessary to achieve the required cutting angle. For the biplanar opening osteotomies the cutting guide has the same radial channels as in the case of uniplanar osteotomy onto which another channel is positioned at a $110^{\circ}$ angle.

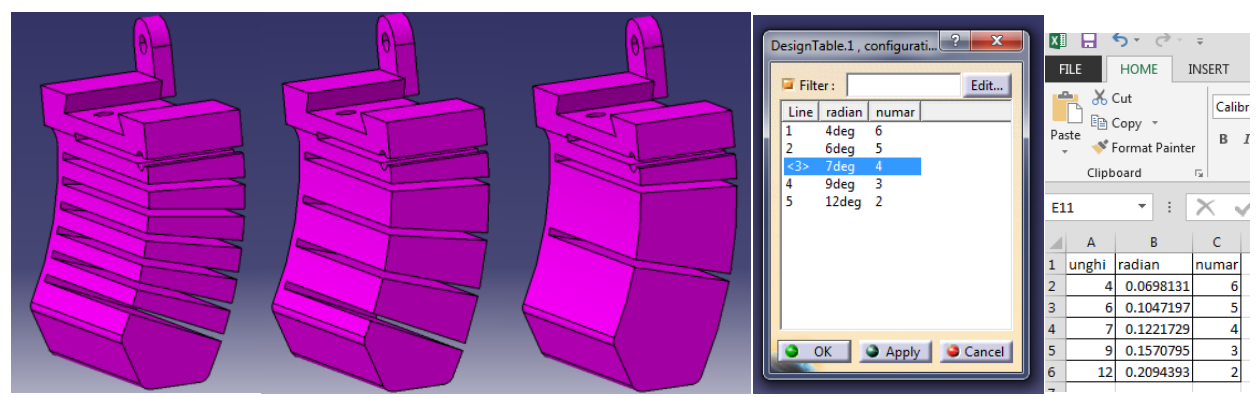

Fig. 9. Parametrisation of the opening osteotomy cutting guide using Excel table data

For even greater flexibility in the design of the device and for a possible customization of it, the active elements such as: cutting guide, bush support plates and drilling bush have been parametrized with the possibility of extracting the parameters from tables or databases. Specifically, in this case the tables used were created in Excel. Thus, dedicated 3D models of different sizes can be obtained by extracting data from these tables in which doctors may have stored information collected from patients or from radiographic images. 
Figure 9 shows the parameterization of the opening osteotomy cutting guide. The parameterized elements are the number of channels of the cutting guide and the angle between them. Five possibilities were considered, three of which are presented in the figure.

\section{Conclusions and further work}

Considering that the High Tibial Osteotomy surgical procedure can be addressed to younger patients for whom total knee prosthesis is not the best solution for the future, the precision and accuracy with which the doctors perform this intervention becomes very important. Surgeries performed without a specialized device reduces the accuracy of the operation to the surgeon's skill, which, however good it may be, is not comparable to the guidance offered by a specialized device. We consider that the modular device described in this paper is a good step toward the adoption of guiding devices during HTO surgeries and its parameterized and modularized design ensures a high degree of generality and applicability for this type of operation.

In the further work we intend to extend our researches to approach the problem regarding the build materials required for this device taking in account the field where it is used. Another interesting research direction is to study the possibility of making customized device components using Additive Manufacturing technologies.

This work is supported by the DiFiCIL project (contract no. 69/08.09.2016, ID P_37_771, web: http://dificil.grants.ulbsibiu.ro), co-funded by ERDF through the Competitiveness Operational Programme 2014-2020.

\section{References}

1. I.I. Cofaru, Researches regarding the biomechanics of the axial deviations of the human lower member and the development of the correspondent surgical devices, $\mathrm{PhD}$ Thesis, Sibiu (2013)

2. AA. Amis, Biomechanics of high tibial osteotomy, Knee Surg Sports Traumatol. Arthrosc. Jan; 21(1), 197-205, (2013)

3. D. Pape, K. Dueck, M. Haag, O. Lorbach, R. Seil, H. Madry A., Wedge volume and osteotomy surface depend on surgical technique for high tibial osteotomy, Knee Surg Sports Traumatology Arthrosc. Jan; 21(1), 127-133 (2013)

4. I.I. Cofaru, Biomechanics of the opening tibial osteotomy, ANNALS OF THE UNIVERSITY OF ORADEA, 23, (2014)

5. I.I. Cofaru, P.D. Brindasu, N.F. Cofaru Designing a Specialized Devices for Correction of the Axis Deviation at the Human Leg, Applied Mechanics and Materials,, (2013)

6. X. Liu, Z. Chen, Y. Gao, J. Zhang, and Z. Jin - High Tibial Osteotomy: Review of Techniques and Biomechanics, Journal of Healthcare Engineering, ID 8363128, (2019)

7. K. Amman, V.P. Novak, R. Schneider, R.E. Burns, United States Application Publication Ammann et al., Apparatus for performing an open wedge, high tibial osteotomy, Patent number US 2015/0057667 A1, Feb. 26, (2015)

8. United States patent Collazo, C.E. Collazo, High tibial osteotomy system, Patent number US8241292B2, Aug.14, (2012)

9. United States patent Collazo, Carlos E. Collazo, High tibial osteotomy instrumentation, Patent number US8192441B2, Jun. 5, (2012)

10. United States patent - Schreiber et al, patent number 5078719, Jan7, (1992)

11. United States patent - Jenkins et al, patent number 5722978, Mar3, (1998) 